\title{
VIEWPOINT
}

\section{What the Marine Mollusc Aplysia Can Tell the Neurologist About Behavioral Neurophysiology}

\author{
PETER RUBEN, JEFF GOLDBERG, JON EDSTROM \\ KAREN VOSHART and KEN LUKOWIAK
}

From the Department of Medical Physiology, Faculty of Medicine, University of Calgary, Calgary, Alberta.

Requests for Reprints to: Dr. K. Lukowiak, Dept. of Medical Physiology, Faculty of Medicine, University of Calgary, Calgary, Alberta, Canada T2N 1 N4.

\section{INTRODUCTION}

Only recently has man begun to regard himself as mundane and not divine. This conceptual liberation has allowed him to ask frank questions concerning the physical and chemical mechanisms which determine or affect his behavior. Unfortunately the answers to these questions have been slow in coming. The reasons for this are two-fold: Basic ethical considerations preclude the experiments necessary to investigate the neural substrates of human behavior in man. Further, man's behavior and nervous system are both so enormously complex and subtle, it is therefore unlikely that much real fundamental knowledge could be gained from such experiments if performed. It is more expedient to study simple behavior in simpler organisms than man to understand how nervous systems operate in general and, it is hoped, to eventually gain a better understanding of the human in particular. This tactic is known as the "model systems" approach. By discovering the strategies adopted by less complex nervous systems to deal with simple situations one can devise a realistic model of the neural mechanisms that control more complex behavior in more advanced animals.

Many animals have served as valuable sources of model systems. Among them the marine gastropod mollusc Aplysia has received considerable attention. In comparison to the human nervous system with approximately 50 billion neurons, the Aplysia nervous system contains relatively few neurons - about 20,000. Furthermore the study of the Aplysia nervous system has several other advantageous char- acteristics. A number of forms of behavioral plasticity that are found in all higher metazoans including man are also found in the Aplysia. These simple but non-trivial types of behavioral plasticity include habituation, sensitization and associative learning as well as easily defined qualities of neural function which we choose to call "behavioral states". In addition the nervous system is composed of neurons which are large and, in many cases, easily identified by anatomical and physiological criteria so that the "same" cell can be studied in more than one animal under more than one set of experimental conditions. The cell bodies of the neurons in Aplysia, from which electrical recordings can be fairly easily obtained, are electrically close to their dendrites so that changes in postsynaptic potentials occurring during modifications of behavior can be monitored.

However, before beginning an examination of the Aplysia model system it is first necessary to consider whether or not the reductionist approach is valid. Can we really expect to uncover the mysteries of the human mind by investigating the brain of a slug? The view of many invertebrate neurobiologists is that it is less important at this time to show how the human brain is special or unique than it is to find the behaviors and their neural mechanisms which are common to man and the model systems (see Kandel 1979). It may be that for each trait common to the human nervous system and model system, a common principle of neural organization or function may be found. The general principles which we learn from the simple system may 
lead us to a better understanding of how our own nervous system works. Our arguments in support of the model system approach are based in large part on the observation that there are no fundamental structural or functional differences between nerve cells and synapses of man and those of slugs.

In this review we shall focus on the model system which consists of the siphon, mantle, gill and abdominal ganglion of the sea slug Aplysia. This collection of organs can be removed from the intact animal (see figure 1) and still behave much as it did in the whole animal. The gill is the main respiratory organ of the Aplysia and contains three sets of muscle which allow it to contract. Periodically it contracts spontaneously to promote blood flow through the gill. These

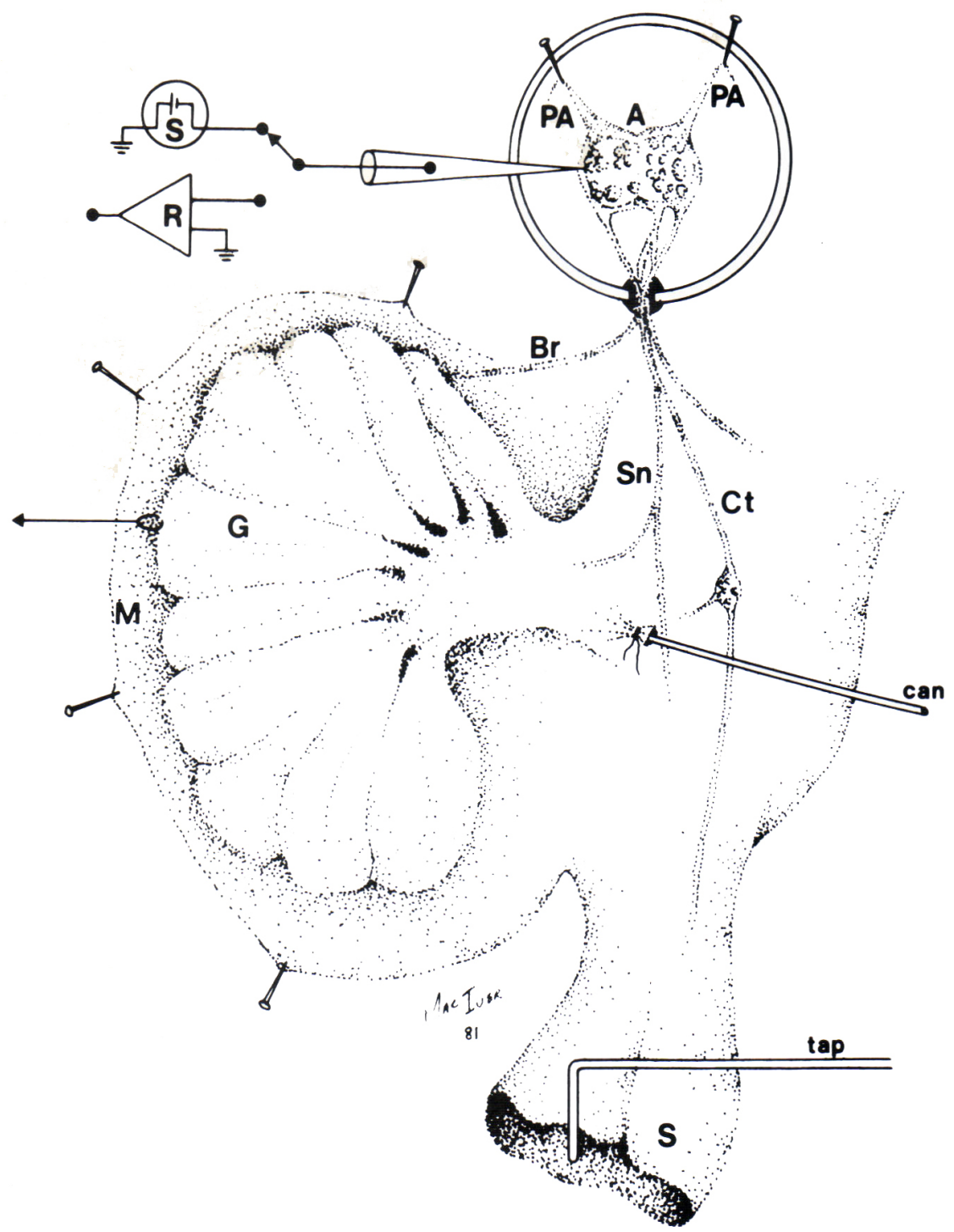

Figure 1-Diagram of experimental preparation. The semi-intact Aplysia californica preparation consists of the siphon (S), mantle (M), gill (G) and abdominal ganglion (A). The gill is innervated from the abdominal ganglion by the branchial $(B)$, ctenidial $(\mathrm{Ct})$ and siphon (Sn) nerves. These nerves were passed under the lower edge of the plastic chamber which surrounds the abdominal ganglion. Also shown are the pleuroabdominal connective nerves (PA). The gill was connected to an isotonic tension transducer. Intracellular recordings were made from identified neurons in the ganglion. A suction electrode was placed on a posterior gill pinnule to record (putative) excitat ory synaptic potentials evoked by identified gill motor neurons. (Ruben, 1981). movements are called spontaneous gill movements (SGM). The gill will also contract and withdraw within the mantle cavity for protection as a reflex to various external stimuli. This is known as the gill withdrawal reflex (GWR) (see figure 2) and is the center of our research and the subject of this review.

The GWR is elicited by applying a tactile stimulus to the siphon. The magnitude of the response of the gill to a constant siphon stimulus is variable and the means by which it is modified can be characterized as associative or non-associative types of learning. Nonassociative learning is defined as a change in behavior following the repetitive presentation of a given stimulus and accounts for habituation, dishabituation and sensitization. Associative learning is defined as the aquisition of a response to a previously neutral stimulus. Here we will refer
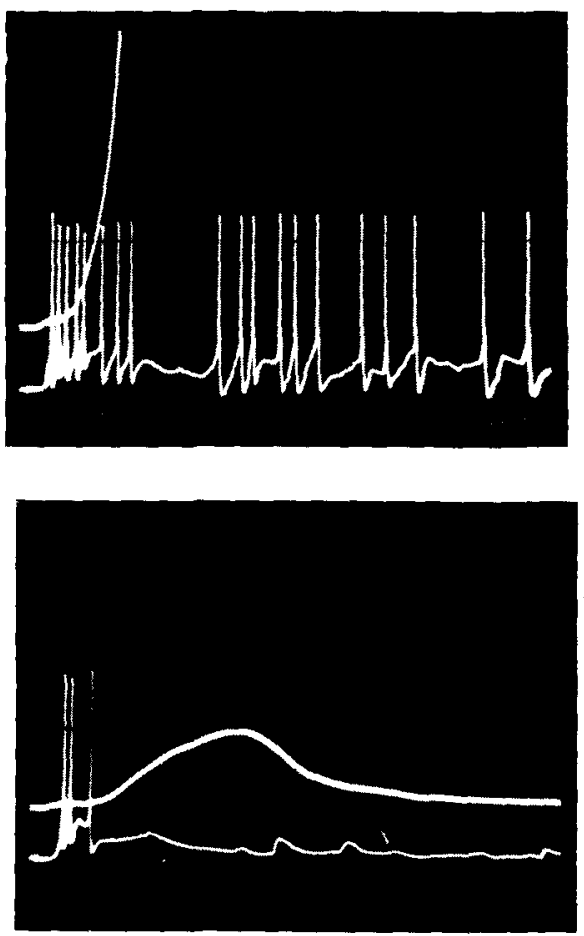

Figure 2-Habituation of the gill withdrawal reflex in Aplysia. Trial 1 is shown in the upper panel. The top trace is the GWR whilst the bottom trace is an intracellular recording from central gill motor neuron L7. Trial 10 is shown in the lower panel. The siphon was stimulated once every 30 seconds with a force of $\mathrm{lg}$. As can be seen, both the GWR amplitude and the number of action potentials evoked in $\mathrm{L} 7$ decrement. 
specifically to classical or "Pavlovian"-learned reflexes. These adaptive changes in behavior are part of the behavioral repertoire common to higher organisms and to the noble sea slug. The remainder of this review will discuss what has been learned from the Aplysia about these important adaptive neural functions.

\section{HABITUATION}

The first form of non-associative learning to consider is habituation. Habituation is a decrement in the amplitude of a response which occurs when a stimulus is presented several times. It is probably the most ubiquitous form of behavioral modification, occurring in the human and aplysian nervous systems. Repetitive stimulation of the Aplysia siphon leads to habituation of the GWR. The reflex and its habituation are mediated by the integrated activity of the central nervous system (CNS) and the peripheral nervous system (PNS). In the CNS (in this context CNS refers to the abdominal ganglion) lies the greatest advantage of the Aplysia preparation: the large identifiable neurons which can be easily impaled with microelectrodes. The neurons in the PNS are not as accessible to electrophysiological investigation but there is evidence that similar mechanisms are at work in the PNS to bring about habituation. From work on habituation in the Aplysia CNS, habituation is now thought to be the result of a decrease in the efficacy of pre-existing synaptic connections between primary sensory neurons and central gill motor neurons (Klein et. al., 1980). The low frequency depression of the monosynaptic connection between the sensory neuron and the motoneuron underlying habituation appears to be an intrinsic property of the sensory neuron and does not require any interneuron activity. The response decrement during habituation is a consequence of a decrease in the amount of neurotransmitter released by the sensory neuron with each successive action potential. Available evidence indicates that the decrease in transmitter release is due to a depression in the inward calcium current in the presynaptic sensory terminal during each action potential. Since less calcium enters with each potential less transmitter will be released. As less transmitter is released the amplitude of the postsynaptic potential will also decrease. As a result, fewer action potentials will be evoked in the motoneuron for each successive stimulus so that the gill withdrawal reflex will decrease in amplitude. A simple behavioral modification, habituation is therefore probably due to a change in an ionic current (calcium) occurring at a specific locus in the nervous system.

\section{SENSITIZATION}

Another form of non-associative learning which occurs in this preparation is sensitization. In contrast to habituation which can be a homosynaptic process, sensitization appears to be a heterosynaptic process. Sensitization is a result of the activity of other neurons' actions on the synaptic terminations of the sensory neurons to produce presynaptic facilitation. This leads to an increased release of transmitter from the sensory neuron's presynaptic terminals. There is now convincing evidence that this presynaptic facilitation is mediated by serotonergic neurons (Klein et al., 1980). Application of exogenous serotonin increases the content of cyclic AMP in the abdominal ganglion and mimics the changes in synaptic transmission observed during sensitization. Injection of cyclic AMP into the sensory neuron also facilitates transmission to the motoneurons. The specific result of increased intracellular cyclic AMP in the sensory neuron is a decrease in the outward potassium current during an action potential. This in turn prolongs the action potential of the sensory cell. During the longer action potential more calcium is allowed to enter so that more transmitter is released during each action potential. As a consequence of this chain of events the postsynaptic potential is increased in magnitude and causes an increased GWR.

Simple non-associative learning in Aplysia has been shown to result from push-pull modulation of presynaptic calcium current by several sources. It remains to be seen if these same biochemical mechanisms are also employed by more complex animals. But it can be seen that the model system approach has provided us with definite hypotheses concerning these adaptive processes which suggest specific experimental approaches to the same phenomena in more complex organisms.

\section{BEHAVIORAL STATES AND REFLEX RESPONSIVENESS}

Over the years we have observed considerable variation in the response to standard siphon stimuli used to elicit the GWR. Some preparations show only a weak response while others show an exceptionally vigorous one. Those preparations exhibiting a weak response habituate rapidly while those which are exceptionally responsive habituate slightly or not at all. We believe that these variable levels of responsiveness reflect the general responsive state of the animal and we refer to these as arousal levels. Three levels of responsiveness have been observed and are referred to as the aroused, suppressed and the normal levels. These levels are operationally defined by comparing the amplitude of the GWR to the amplitude of the SGM.

To date, the suppressed level has been correlated with two behaviors: feeding and sexual activity. Suppression of the GWR can also be induced in a normal preparation by pharmacological manipulations. Feeding and sexual behavior comprise a large portion of the Aplysia's behavioral repertoire, and adult Aplysia do not have any natural predators (except neuroscientists). It seems reasonable that a defensive reflex such as the GWR should be suppressed once the animal's major biological requirements have been met.

Whether or not this teleological explanation is correct, we have begun to uncover the neuromechanisms which bring about the suppression. Possible mechanisms underlying both behavioral states and the operationally defined arousal level have been suggested (Thornhill et al., 1981). 


\section{PHARMACOLOGY OF SUPPRESSION}

As mentioned above, we have been able to alter the level of responsiveness of a normal preparation to that of a suppressed one. We have shown that suppression can be induced by bathing the abdominal ganglion in a saline solution of Arginine vasotocin (AVT) or met-enkephalin (m-ENK). These two peptides show a potent suppressive effect in picomolar concentrations. The actions of AVT and $\mathrm{m}-\mathrm{ENK}$ in Aplysia are fairly characteristic of those reported elsewhere for putative peptide neurotransmitters in that their actions are slow in onset (approximately 10 minutes) and long lasting (about 90 minutes). We may be able to better understand how peptides exert their effects in a mammalian nervous system by examining the manner in which they operate in Aplysia. For example in mammals, enkephalin modulates synaptic transmisssion between sensory neurons and interneurons in the spinal cord. Unfortunately, detailed examination of the mechanism of this modulation has only been possible in primary tissue culture where the degree of applicability to in-vivo conditions is questionable.

When AVT or m-ENK is superfused over the abdominal ganglion, they suppress the GWR and decrease the number of action potentials evoked in gill motoneurons by tactile siphon stimulation. Suppression of the GWR in both the natural and pharmacologically suppressed preparations might be due to either a reduction in the response of the sensory neuron to a given stimulus or by a direct effect on synaptic transmission between the sensory neuron and the motoneuron. By simultaneously recording from a representative sensory neuron and a motoneuron we have been able to demonstrate that AVT and m-ENK do not significantly affect the excitability of the sensory neurons and they do not affect the passive membrane properties of the gill motoneurons. The peptides exert their effect by decreasing the size of the EPSP (Goldberg and Lukowiak, 1981). This suggests that they modulate release of neurotransmitter from the sensory neuron. Thus, some behavioral states may not be exclusively caused by a monosynaptic process, but may also be influenced by heterosynaptic mechanisms as well. Experiments are now in progress to determine how the peptides affect transmitter release in Aplysia.

Although the superfusion of AVT over the abdominal ganglion alters the responsiveness of the preparation one must question the significance of the observation. Recently we have been able to demonstrate that AVT is endogenous to the Aplysia CNS. We have yet to demonstrate that the naturally occurring suppressed behavioral state is mediated by the release of AVT in the abdominal ganglion although evidence is beginning to accumulate in support of this hypothesis.

We have been able to pharmacologically differentiate between two naturally occurring suppressed behavioral states. Naloxone, an opiate antagonist, relieves the suppression seen in a preparation taken from a sexually active animal but does not affect the suppression observed in food satiated animals. At the cellular level we have found that in preparations taken from sexually active animals, an action potential in a siphon sensory neuron does not evoke an easily observable EPSP in gill or siphon motoneurons. After application of naloxone a larger EPSP is observed in the postsynaptic neuron following a presynapyic action potential. Naloxone does not affect the monosynaptic EPSP amplitude in the same way in preparations taken from food satiated animals. Thus, it may be that two different peptidergic systems mediate behavioral suppression; an AVT pathway subserving behavioral modifications following feeding and an opiate pathway subserving the behavioral modifications following sexual activity.

\section{PHARMACOLOGY OF AROUSAL}

The aroused behavioral state can also be simulated pharmacologically (Ruben, 1981). Although the natural causes of the aroused state are not known, the pharmacological substrate is beginning to be explored. The GWR is enhanced in normal preparations if the gill is perfused with dopamine. The GWR amplitude increases and habituation is now blocked. The responsiveness of a normal preparation can also be enhanced electrophysiologically by increasing the tonic firing rate of motor neuron L9. While L9 firing is enhanced, the reflex amplitude increases and becomes resistant to habituation. When the gill is perfused by dopamine receptor blockers such as ergonovine and chlorpromazine, L9 activity is no longer able to affect the amplitude of the GWR. Furthermore, in naturally aroused preparations perfusion of the gill with the same dopamine antagonists reverses the aroused behavioral state and the preparation behaves normally.

The effects of dopamine on the responsiveness of the GWR only appear when the drug is perfused through the gill; no effect on GWR is seen when the drug is applied directly to the CNS alone. Through a series of experiments designed to differentiate between possible sites of action in the gill it was found that dopamine's action is probably restricted to the peripheral terminations of central motoneurons. More specifically, it was found that dopamine probably only affects the terminals of the motoneuron L7. What is more, dopamine seems only to affect the terminations of one branch of L7's axon. Gill contractions caused by the ctenidial branch of $\mathrm{L} 7$ are facilitated by perfusion of the gill by dopamine while the contractions caused by the branchial branch of L7's axon are not. Presumably dopamine affects either the release of transmitter from L7's ctenidial branch or it enhances the excitationcontraction coupling of the muscles activated by this branch of L7's axon.

\section{CLASSICAL CONDITIONING}

Experiments have begun which may enable us to elucidate the neural mechanisms underlying simple associative learning. We have succeeded in classically conditioning the GWR in the in-vitro preparation. The Aplysia siphon contains photoreceptors, and under normal circumstances photic stimulation elicits a contraction of the 
siphon but no gill movement. A strong tactile stimulation of the gill causes contraction of both the siphon and the gill. By pairing photic stimulation of the siphon with tactile stimulation of the gill, with the photic stimulus preceeding the tactile stimulus, the preparation will, after several paired presentations of the two stimuli, begin to respond to the photic stimulus by contracting the gill in addition to the siphon. As the acquired reflex movement of the gill to photic stimulation develops, there is a concomitant increase in the light-induced synaptic activity observed in gill motoneurons. It appears that classical conditioning either increases the efficacy of weak pre-existing synaptic pathways or it induces the formation of novel synaptic connections. At present our hypothesis is that the photosensory pathway to the gill motoneurons already exists but is either ineffective or nonfunctional under normal circumstances. With training the strength of the synaptic pathway is increased.

\section{SUMMARY AND CONCLUSIONS}

From the above brief description of current work in the Aplysia conclusions can be drawn at two levels. We can see where we stand in the present, look ahead to see what directions research may take in the near future and attempt to predict what lurks around the corner.

Inspection of the relatively simple non-associative forms of learning, habituation and sensitization, has revealed that in Aplysia these processes are probably controlled by altering the amount of calcium entry into sensory nerve terminals so that the amount of transmitter released with each afferent impulse will increase or decrease depending on whether habituation or sensitization is taking place. It was also shown that the two opposing functions are dependent on different neural mechanisms. Habituation was seen to be a homosynaptic phenomenon while sensitization was shown to be a heterosynaptic process that required the activity of at least one other interneuron.

We also saw that as simple as it may first appear, the Aplysia is capable of exhibiting startling differences in responsiveness which we have called behavioral state and which may be analagous to arousal and motivation in higher animals. The natural origins of these behavioral states are partially clear at this time, and it was shown that the simplicity of the system has allowed a direct and detailed pharmacological analysis to begin. This investigation has revealed that the suppressed behavioral state may well be determined by the release of peptide transmitters or hormones and that there are at least two putative peptide transmitters to consider, AVT and $\mathrm{m}$ ENK, both of which are found in higher vertebrates. In addition it was tentatively hypothesized that although both peptides interfere with neurotransmission at a presynaptic site, they probably mediate suppression independently in response to two different physiological stimuli, sexual activity and feeding.

The nature of the aroused state was also discussed and seen to be a specific heterosynaptic enhancement of the efficacy of the efferent and not the afferent system. Moreover it was shown that the aroused state was mediated by one nerve in particular. Arousal, it seems, is a very specific mechanism which can fine tune an evolutionarily persistent and stable machine.

The simple reduced model system was also shown to be an ideal vehicle with which to approach the challenge of investigating associative learning. This is truly in its infancy but already has strongly indicated in which direction future research should be directed. All that has been learned so far is that it is possible to observe it in the reduced Aplysia preparation.

The next obvious question is "Whither the Aplysia"? Two paths can be seen to diverge from the point at which we stand today. On the one hand we can continue to investigate the neurophysiology of the Aplysia. By so doing we can extend our understanding of the phenomena described in the above review. For example, now that the substrate of habituation has been tentatively identified the process can be more fully described and investigated so that effective and controlled pharmacological intervention in the process may become possible. Alternative mechanisms may be uncovered in other sensory or integrative circuits and, most interesting of all, the manner in which all of the known neural mechanisms interact to form complex intact behavior can be explored in hopes of discerning how emergent properties of neural networks are formed.

On the other hand the information gained from working on model systems will be applied to higher organisms. Initially this will only be used with more complex but still simple model systems like frogs, cats, and cell or tissue culture. In this process the concepts, techniques and talents derived from the investigation of model systems will almost certainly be expanded, refined or discarded. Even so the preceeding work in model systems will have served the purpose of at least allowing us to ask the higher organism intelligent questions.

Although we may be well on the way to understanding some simple forms of behavior in model systems we have a long way yet to go before the mysteries of the human mind stand revealed. It is a long road indeed that leads from aplysian behavioral states to the dazzling array of palsies, paranoias and psychoses which man is heir to. As if that were not enough, once these issues are resolved we must one day confront the problem of finding the neural substrate of ESP, clairvoyance and psychokinesis. While mankind lurches menacingly into the future we, of the optomistic viewpoint, like to think that one day he will eventually come to understand the neural substrates of behavior.

This work is supported by the Medical Research Council of Canada (KL,JE) and Alberta Heritage Foundation for Medical Research Studentship awards (PR, JG, KV).

\section{REFERENCES}

GOLDBERG, J., and LUKOWIAK, K., (1981). Modulation of a sensory-motor synapse by neuroactive peptides in Aplysia californica. Soc. Neurosci. 7: 160. 
KANDEL, E.R., (1979). Cellular insights into behavior and learning. The Harvey Lectures, 1979, Academic Press.

KLEIN, M., SHAPIRO, E., and KANDEL, E., (1980). Synaptic plasticity and the modulation of the $\mathrm{Ca}^{2+}$ current. J. Exp. Biol. 89: 117-157.
LUKOWIAK, K., and SHALEY, C., (1981). The In Vitro classical conditioning of the gill withdrawal reflex in Aplysia. Science 212: 1516-1518.

RUBEN, P., (1981). Dopamine mimicry of aroused gill reflex behaviors in Aplysia Californica. PhD Thesis, University of Calgary 1981.
THORNHILL, J., LUKOWIAK, K., COOPER, K., VEALE, W., and EDSTROM, J., (in press): Arginine vasotocin, an endogenous neuropeptide of Aplysia, supresses the gill withdrawal reflex and reduces the evoked synaptic input to central gill motor neurons. J. Neurobiol. 